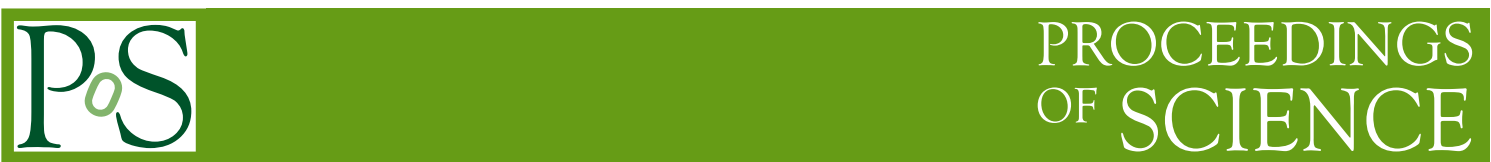

\title{
Multi-Loop Calculations in the MSSM
}

\author{
Luminita Mihaila ${ }^{* \dagger}$ \\ Institut für Theoretische Teilchenphysik, Universität Karlsruhe, Karlsruhe Institut of Technology \\ (KIT), 76218 Karlsruhe, Germany \\ E-mail: luminita@particle.uni-karlsruhe.de
}

In this talk we report on recent high precision calculations within the minimal supersymmetric extension of the Standard Model (MSSM). In the first part, methods and techniques appropiate to the multi-loop calculations in supersymmetric theories using the component field approach are presented. As an example, we discuss the most accurate prediction of the strong coupling constant at very high energies like the Grand Unification(GU) scale. The last part of the talk allude to the calculation of the three-loop corrections to the light CP-even Higgs boson mass taking into account effects from SUSY-QCD.

RADCOR 2009 - 9th International Symposium on Radiative Corrections (Applications of Quantum Field Theory to Phenomenology),

October 25 - 302009

Ascona, Switzerland

\footnotetext{
* Speaker.

${ }^{\dagger}$ Thanks to R. Harlander, P. Kant, J. Salomon and M. Steinhauser for collaboration on the work presented here.
} 


\section{Introduction}

After two decades or more of testing the Standard Model (SM) predictions through collider experiments, no clear evidence for physics beyond the Standard Model (BSM) has been found. Yet there is a strong belief that the SM cannot be the ultimate theory, as it cannot explain the dark matter and the dark energy, it requires fine-tuning to stabilize the Higgs boson mass upon radiative corrections and does not predict unification of the gauge couplings. Theories based on Supersymmetry (SUSY) are among the most appealing extensions of the SM, as they provide elegant solutions to all these questions and are highly predictive. So far, the direct search for SUSY particles have only set lower bounds of about $100 \mathrm{GeV}$ on their masses. However, the next generation of high-energy experiments at the Large Hadron Collider (LHC) and the International Linear Collider (ILC) will allow precision tests of the theoretical models predicting SUSY particles at the TeV scale. Apart from the direct searches, another possibility to probe SUSY is to study the quantum effects generated by the SUSY particles on the high precision observables. Among the most sensitive observables to SUSY radiative corrections (that require at least two-loop order corrections to cope with the experimental accuracy) are the mass of the lightest CP-even Higgs boson, the rho parameter, the muon anomalous moment and the $b \rightarrow s \gamma$ branching ratio.

Another compelling argument in favour of SUSY is the particle content of the MSSM that leads in a natural way to the unification of the three gauge couplings at a high energy scale $\mu \simeq$ $10^{16} \mathrm{GeV}$, in agreement with predictions of Grand Unification Theories (GUT). It is often argued ( see e.g. Refs. [1]) that, from the precise knowledge of the low-energy supersymmetric parameters one can shed light on the origin and mechanism of supersymmetry breaking and even on physics at much higher energies, like the GUT scale. The extrapolation of the supersymmetric parameters measured at the $\mathrm{TeV}$ energy scale to the GUT-scale raises inevitably the question of uncertainties involved.

In this paper, we report on the prediction of two physical observables that require even the three-loop SUSY radiative corrections. Namely, we present in section 3 the unification of the coupling constant within the MSSM and in section 4 the prediction of the lightest Higgs boson mass within the MSSM.

\section{Framework}

The precision tests of SUSY models require both high precision measurements and theoretical calculations. The later ones comprise usually multi-loop or multi-leg computations, which necessarily raise the question of the choice of regularization scheme. The precision calculations performed within the SM are dominated by the dimensional regularization (DREG) scheme, due to its elegant feature to maintain the gauge invariance. DREG is, however, less well-suited for SUSY theories because invariance of a given action with respect to SUSY transformations only holds in general for specific values of the space-time dimension $D$. An elegant way to modify the DREG so as to render it compatible with SUSY was proposed by Siegel [2]. Namely, the continuation from 4 to $D$ dimensions is made by compactification. After dimensional reduction to $D=4-2 \varepsilon$, it is only the first $D$ components of the gauge field that generate the actual gauge interactions. The remaining $2 \varepsilon$ components behave under gauge transformations as a multiplet of scalar fields, usually 
called $\varepsilon$-scalars. In contrast to the SUSY theories, in softly broken supersymmetric theories like the MSSM, the $\varepsilon$-scalars receive a loop-induced mass, which will also influence the renormalization of the genuine scalar masses. In order to decouple the $\varepsilon$-scalar masses from the $\beta$-functions of the genuine scalar masses, additional finite counterterms proportional to the $\varepsilon$-scalar masses have to be added to the renormalized scalar masses $[3,4]$.

There are potential problems even within DRED. If the variation $\delta S$ of the action gives a nonzero result when inserted in a Green's function this creates an apparent violation of supersymmetric Ward identities. All explicit calculations up to two-loop order have found zero for such insertions $[5,6]$. Another way to verify the consistency of DRED with SUSY is to study the behaviour under the renormalisation of the $\varepsilon$-scalar-couplings (also called evanescent couplings) to matter and gauge fields. In a supersymmetric theory, they have to remain equal to the gauge coupling, if the renormalization scheme preserves SUSY. Explicit computations up to three-loop order within SUSY-QCD [7] confirmed this requirement for DRED in combination with the minimal subtraction scheme, i.e. the $\overline{\mathrm{DR}}$ scheme.

\section{Coupling constant unification}

In this section we present the numerical impact of the three-loop order SUSY-QCD corrections on the prediction of $\alpha_{s}\left(\mu_{\mathrm{GUT}}\right)$. This prediction is obviously directly related to the scale and quality of unification of the gauge couplings.

It is well known that the equality of the Yukawa couplings of gauginos to matter multiplets and the gauge couplings, or the equality of the quartic scalar couplings, e.g. four-squark or fourslepton couplings, and the gauge couplings are not preserved under renormalization if DREG is employed. This point becomes important if we want to relate a given theory at one scale to the same theory at another scale. In general the SM parameters and cross sections are mostly given in the $\overline{M S}$ scheme, while the MSSM ones are usually given in the $\overline{\mathrm{DR}}$ scheme. Apart from the finite shifts $[8,9]$ of the running parameters associated with the change of renormalization scheme, also threshold corrections $[10,11]$, which account for the non-decoupling of heavy particles in mass independent schemes have to be taken into consideration.

For the energy evolution of the strong coupling we follow the method proposed in Ref. [12, 13]: first, we compute $\alpha_{s}^{(5)}\left(\mu_{\mathrm{dec}}\right)$ from $\alpha_{s}^{(5)}\left(M_{Z}\right)$ using the corresponding $i$-loop SM RGEs [14]. Here $\mu_{\mathrm{dec}}$ denotes the energy scale at which the heavy particles are supposed to become active, i.e. the scale where the matching between the SM and the MSSM is performed. For consistency, the $i$-loop running parameters have to be folded with $(i-1)$-loop conversion and decoupling relations. Above the decoupling scale, the energy dependence of the running parameters is governed by the $i$-loop MSSM RGEs [15, 7]. We solved numerically the system of coupled differential equations arising from the two sets of RGEs, and implemented this procedure for $i=1,2,3$.

The decoupling scale is not a physical parameter and cannot be predicted by the theory. It is usually chosen to be of the order of the heavy particle mass in order to circumvent the appearance of large logarithms. At fixed order perturbation theory, it is expected that the relations between the running parameters evaluated at high-energy scales and their low-energy values become less sensitive to the choice of $\mu_{\mathrm{dec}}$ once higher order radiative corrections are considered. The dependence 
on the precise value of the decoupling scale is interpreted as a measure of the unknown higher order corrections.

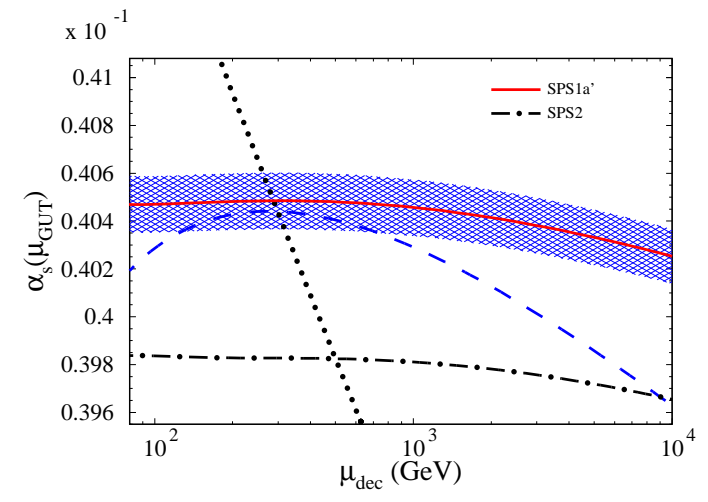

Figure 1: $\alpha_{s}\left(\mu_{\mathrm{GUT}}\right)$ as a function of $\mu_{\mathrm{dec}}$. Dotted, dashed and solid lines denote the one-, two-, and three-loop contributions obtained using as input the SPS1 ${ }^{\prime}$ point. The dash-dotted line shows the threeloop running corresponding to the SPS2 point.
The dependence on the decoupling scale for $\alpha_{s}\left(\mu_{\mathrm{GUT}}\right)$ is displayed in Fig. 1. The dotted, dashed and solid lines denote the one-, two-, and three-loop running, where the corresponding exact results for the decoupling coefficients have been implemented. One can see the improved stability of the threeloop results w.r.t. the decoupling-scale variation. The uncertainty induced by the current experimental accuracy on $\alpha_{s}\left(M_{Z}\right), \delta \alpha_{s}=$ 0.001 [16], is indicated by the hatched band.

In order to get an idea about the effects induced by the SUSY mass parameters on $\alpha_{s}\left(\mu_{\mathrm{GUT}}\right)$, we show also the results for the Snowmass Point SPS1a ${ }^{\prime}$ and SPS2 [17].

One clearly notices the great impact of the SUSY-mass pattern on the predicted value of the strong coupling at high energies. Accordingly, for precision studies the explicit mass pattern of heavy particles must be taken into account.

\section{Higgs boson mass in the MSSM}

The MSSM Higgs sector is very much restricted by SUSY and basically can be described by two independent parameters. The mass of the lightest $\mathrm{CP}$-even Higgs boson, $\mathrm{Mh}$, is not a free parameter anymore, but it can be predicted within the theory. Its strong sensitivity to radiative corrections is notorious. In the lowest order it is bounded from above by the $\mathrm{Z}$ boson mass which is already excluded by experiment. But it has been observed that large one-loop corrections, in particular from the top quark and squark sector can raise $M_{h}$ to about $140 \mathrm{GeV}$ [18]. From the knowledge of the exact one-loop corrections [19] one can learn that the dominant effects can be obtained in the so-called effective-potential approximation, namely, the limit of vanishing external momentum. All relevant two-loop corrections have been evaluated with very few exceptions in this approach (for reviews, see Refs. [20]). They can reach as much as $20 \mathrm{GeV}$ and have opposite sign as compared with the one-loop contributions. The currently available three-loop order SUSY-QCD corrections [21, 22] sum up to few GeV. This has to compared with the expected experimental resolution of about $100-200 \mathrm{MeV}$ for the LHC [23] and of roughly $50 \mathrm{MeV}$ for the ILC [24]. This clearly indicates the necessity of the calculation of three-loop order corrections.

We performed the evaluation of the three-loop order corrections within the diagrammatic approach. Due to the presence of many mass scales, an exact evaluation of the three-loop integrals is out of range. However it is possible to apply expansion techniques for various limits, which allows to cover almost the complete SUSY parameter space. For the renormalization scheme we adopted 
the $\overline{\mathrm{DR}}$ scheme for the top quark mass, the masses of the SUSY particles and the top mixing angle. The choice of $\overline{\mathrm{DR}}$ scheme instead of the on-shell one for the top quark mass renormalization improves significantly the convergence of the perturbation series for $M_{h}$. This is a well known fact and can be explained by the absence of large contributions originating from the infra-red sector specific for the on-shell masses.

As an example for the three-loop effects, we show below the prediction for $M_{h}$ as a function of the parameter $M_{1} / 2$ for the benchmark scenario SPS2.

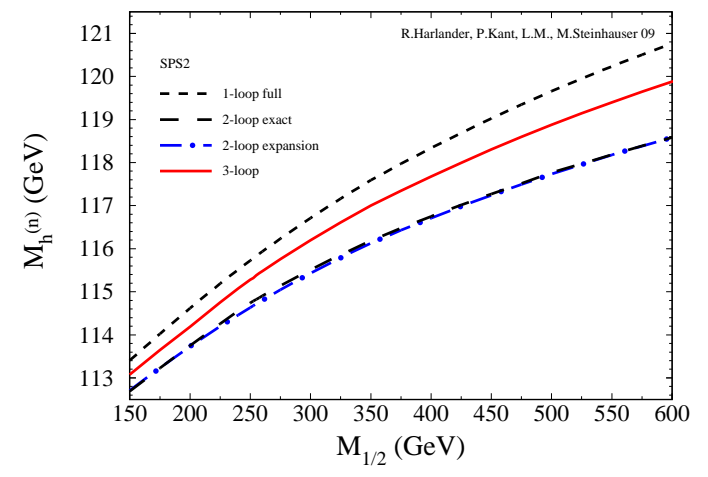

Figure 2: $M_{h}$ for the benchmark scenario SPS2. Dashed, dash-dotted and solid lines corresponds to the one-, two- and three-loop predictions. The lines with longer dashes correspond to the exact two-loop results.
In Fig. 2, the predictions for $M_{h}$ are shown, where the dashed, dash-dotted and solid lines corresponds to the one-, two- and three-loop results. For comparison, the twoloop exact results are depicted with longer dashes. One clearly notices the very good agreement between the exact and the asymptotically expanded results at two-loop level. One also observe negative corrections between 1 and $4 \mathrm{GeV}$ at two-loops and positive contributions from the three-loop term which amounts to about $2 \mathrm{GeV}$.

Let us mention once again the reduced magnitude of the radiative corrections at all orders, when one uses the $\overline{\mathrm{DR}}$ scheme instead of the on-shell scheme. On the other hand the magnitude of the three-loop order corrections are almost an order of magnitude larger than the experimental accuracy expected to be reached at the LHC, that render them indispensable for phenomenological analysis.

\section{Acknowledgements}

This work was supported by the DFG through SFB/TR 9.

\section{References}

[1] G. A. Blair, W. Porod and P. M. Zerwas, Eur. Phys. J. C 27 (2003) 263 [arXiv: hep-ph/0210058].

[2] W. Siegel, Phys. Lett. B 84 (1979) 193.

[3] I. Jack, D. R. T. Jones, S. P. Martin, M. T. Vaughn and Y. Yamada, Phys. Rev. D 50 (1994) 5481 [arXiv: hep-ph/9407291].

[4] S. P. Martin, Phys. Rev. D 65 (2002) 116003 [arXiv: hep-ph/ 0111209 ].

[5] I. Jack and D. R. T. Jones, In *Kane, G.L. (ed.): Perspectives on supersymmetry* 149-167 [arXiv: hep-ph/9707278]. 
[6] D. Stöckinger, JHEP 0503 (2005) 076 [arXiv : hep-ph/ 0503129$].$

[7] R. V. Harlander, L. Mihaila and M. Steinhauser, Eur. Phys. J. C 63 (2009) 383 [arXiv: 0905.4807 [hep-ph]].

[8] S. P. Martin and M. T. Vaughn, Phys. Lett. B 318 (1993) 331 [arXiv: hep-ph/9308222].

[9] L. Mihaila, Phys. Lett. B 681 (2009) 52 [arXiv:0908.3403 [hep-ph] ].

[10] D. M. Pierce, J. A. Bagger, K. T. Matchev and R. J. Zhang, Nucl. Phys. B 491 (1997) 3 [arXiv: hep-ph/9606211].

[11] A. Bauer, L. Mihaila and J. Salomon, JHEP 0902 (2009) 037 [arXiv: 0810.5101 [hep-ph] ].

[12] R. Harlander, L. Mihaila and M. Steinhauser, Phys. Rev. D 72 (2005) 095009 [arXiv: hep-ph/0509048].

[13] R. V. Harlander, L. Mihaila and M. Steinhauser, Phys. Rev. D 76 (2007) 055002 [arXiv:0706.2953 [hep-ph]].

[14] M. Steinhauser, Phys. Rept. 364 (2002) 247 [arXiv : hep-ph / 0201075$].$

[15] P. M. Ferreira, I. Jack and D. R. T. Jones, Phys. Lett. B 387 (1996) 80 [arXiv : hep-ph/96054 40 ].

[16] S. Bethke, Prog. Part. Nucl. Phys. 58 (2007) 351 [arXiv: hep-ex/0606035].

[17] N. Ghodbane and H. U. Martyn, in Proc. of the APS/DPF/DPB Summer Study on the Future of Particle Physics (Snowmass 2001) ed. N. Graf, [arXiv: hep-ph/0201233]

[18] J. R. Ellis, G. Ridolfi and F. Zwirner, Phys. Lett. B 257 (1991) 83; Phys. Lett. B 262 (1991) 477; Y. Okada, M. Yamaguchi and T. Yanagida, Prog. Theor. Phys. 85 (1991) 1; H. E. Haber and R. Hempfling, Phys. Rev. Lett. 66 (1991) 1815.

[19] P. H. Chankowski, S. Pokorski and J. Rosiek, Phys. Lett. B 274 (1992) 191.; A. Brignole, Phys. Lett. B 281, 284 (1992); A. Dabelstein, Z. Phys. C 67, 495 (1995) [arXiv:hep-ph/9409375].

[20] S. Heinemeyer, Int. J. Mod. Phys. A 21 (2006) 2659 [arXiv: hep-ph/ 040724 4]; B. C. Allanach, A. Djouadi, J. L. Kneur, W. Porod and P. Slavich, JHEP 0409 (2004) 044 [arXiv:hep-ph/0406166].

[21] S. P. Martin, Phys. Rev. D 75 (2007) 055005 [arXiv: hep-ph/ 0701051 ].

[22] R. V. Harlander, P. Kant, L. Mihaila and M. Steinhauser, Phys. Rev. Lett. 100 (2008) 191602 [Phys. Rev. Lett. 101 (2008) 039901] [arXiv:0803.0672 [hep-ph] ].

[23] CMS Physics TDR, Volume II, http: / / cmsdoc.cern.ch/cms/cpt/tdr/.

[24] J. A. Aguilar-Saavedra et al. [ECFA/DESY LC Physics Working Group], [arXiv: hep-ph/0106315]. 